INPLASY

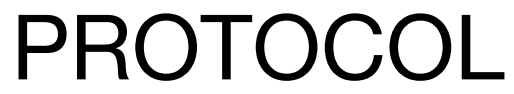

To cite: Li et al. Diagnostic accuracy of ultrasound superb microvascular imaging for lymph nodes: A protocol for systematic review and meta analysis. Inplasy protocol

202070133. doi:

10.37766/inplasy2020.7.0133

Received: 31 July 2020

Published: 31 July 2020

Corresponding author:

Xiukun Hou

fytgzy@163.com

Author Affiliation:

The First Affiliated Hospital of Dalian Medical University

Support: Liaoning Natural Science Found.

Review Stage at time of this submission: Preliminary searches.

Conflicts of interest:

None.

\section{Diagnostic accuracy of ultrasound superb microvascular imaging for lymph nodes: A protocol for systematic review and meta analysis}

\author{
Li, X1; Wang, C2; Hou, X33 Tao, Y4.
}

Review question / Objective: The present meta-analysis aimed at determining the accuracy of superb microvascular imaging in the differential diagnosis between benign and malignant lymph nodes.

Condition being studied: Studies suggested that superb microvascular imaging is helpful for the differentiation between benign and malignant lymph nodes. However, the results of these studies have been contradictory.

Information sources: PubMed, Web of Science, Cochrane Library, and Chinese biomedical databases will be searched from their inceptions to the July 31, 2020, without language restrictions.

INPLASY registration number: This protocol was registered with the International Platform of Registered Systematic Review and Meta-Analysis Protocols (INPLASY) on 31 July 2020 and was last updated on 31 July 2020 (registration number INPLASY202070133).

\section{INTRODUCTION}

Review question / Objective: The present meta-analysis aimed at determining the accuracy of superb microvascular imaging in the differential diagnosis between benign and malignant lymph nodes.
Rationale: As a novel ultrasound technique, superb microvascular imaging can quickly, simply and noninvasively study the microvascular distribution in the tumor and evaluate the microvascular perfusion.

Condition being studied: Studies suggested that superb microvascular imaging is 
helpful for the differentiation between benign and malignant lymph nodes. However, the results of these studies have been contradictory.

\section{METHODS}

Participant or population: Lymphadenopathy.

Intervention: SMI.

\section{Comparator: Pathology.}

Study designs to be included: Clinical cohort or case control studies.

Eligibility criteria: 2.1.1. Type of study. This study will only include high quality clinical cohort or case control studies. 2.1.2. Type of patients. The patients should be those who had undergone Lymphadenopathy. 2.1.3. Intervention and comparison. This study compare SMI with pathology for diagnosing lymph nodes. 2.1.4. Type of outcomes. The primary outcomes include sensitivity, specificity, positive and negative likelihood ratio, diagnostic odds ratio, and the area under the curve of the summary receiver operating characteristic .

Information sources: PubMed, Web of Science, Cochrane Library, and Chinese biomedical databases will be searched from their inceptions to the July 31, 2020, without language restrictions.

Main outcome(s): The primary outcomes include sensitivity, specificity, positive and negative likelihood ratio, diagnostic odds ratio, and the area under the curve of the summary receiver operating characteristic .

Quality assessment / Risk of bias analysis: Methodological quality was independently assessed by two researchers based on the quality assessment of studies of diagnostic accuracy studies (QUADAS) tool. The QUADAS criteria included 14 assessment items. Each of these items was scored as "yes" (2), "no" (0), or "unclear"(1). The QUADAS score ranged from 0 to 28 , and a score $\geq 22$ indicated good quality. Any disagreements between 2 investigators will be solved through discussion or consultation by a 3rd investigator.

Strategy of data synthesis: The STATA version 14.0 (Stata Corp, College Station, TX, USA) and Meta-Disc version 1.4 (Universidad Complutense, Madrid, Spain) softwares were used for meta-analysis. We calculated the pooled summary statistics for sensitivity, specificity, positive and negative likelihood ratio, and diagnostic odds ratio with their $95 \%$ confidence intervals. The summary receiver operating characteristic curve and corresponding area under the curve were obtained. The threshold effect was assessed using Spearman correlation coefficients. The Cochran's Q-statistic and I test were used to evaluate potential heterogeneity between studies. If significant heterogeneity was detected( $Q$ test $P 50 \%)$, a random effects model or fixed effects model was used.

Subgroup analysis: We also performed sub group and meta-regression analyses to investigate potential sources of heterogeneity.

Sensibility analysis: To evaluate the influence of single studies on the overall estimate, a sensitivity analysis was performed.

\section{Country(ies) involved: China.}

Keywords: lymph node; meta-analysis; superb microvascular imaging.

Contributions of each author:

Author 1 - Xuejiao Li.

Author 2 - Cong Wang.

Author 3 - Xiukun Hou.

Author 4 - Ye Tao. 